\title{
VEGF and Ki-67 Overexpression in Predicting Poor Overall Survival in Adenoid Cystic Carcinoma
}

\section{Seongyeol Park, MD 1 \\ Soo Jeong Nam, MD2,3,4 \\ Bhumsuk Keam, MD, PhD ${ }^{1,5}$ \\ Tae Min Kim, MD, PhD 1,5 \\ Yoon Kyung Jeon, MD, PhD2 \\ Se-Hoon Lee, MD, PhD 1,5 \\ J. Hun Hah, MD, PhD ${ }^{6}$ \\ Tack-Kyun Kwon, MD, PhD ${ }^{6}$ \\ Dong-Wan Kim, MD, PhD 1,5 \\ Myung-Whun Sung, MD, $P h D^{6}$ \\ Dae Seog Heo, MD, PhD ${ }^{1,5}$ \\ Yung-Jue Bang, MD, PhD',5}

Departments of ${ }^{1}$ Internal Medicine and ${ }^{2}$ Pathology, Seoul National University

Hospital, Seoul,

${ }^{3}$ The Tumor Immunity Medical

Research Center, Cancer Research Center,

Seoul National University

College of Medicine, Seoul,

${ }^{4}$ Department of Pathology,

National Cancer Center, Goyang,

${ }^{5}$ Cancer Research Institute,

Seoul National University

College of Medicine, Seoul,

${ }^{6}$ Department of Otorhinolaryngology,

Seoul National University Hospital, Seoul, Korea

Correspondence: Bhumsuk Keam, MD PhD Department of Internal Medicine,

Seoul National University Hospital,

101 Daehak-ro, Jongno-gu, Seoul 03080, Korea

Tel: 82-2-2072-7215

Fax: 82-2-2072-7379

E-mail: bhumsuk@snu.ac.kr

Received March 4, 2015

Accepted May 13, 2015

Published Online July 14, 2015

*Seongyeol Park and Soo Jeong Nam contributed equally to this work.

\section{Purpose}

The purpose of this study was to evaluate potential prognostic factors in patients with adenoid cystic carcinoma (ACC).

\section{Materials and Methods}

A total of 68 patients who underwent curative surgery and had available tissue were enrolled in this study. Their medical records and pathologic slides were reviewed and immunohistochemistry for basic fibroblast growth factor, fibroblast growth factor receptor (FGFR) 2 , FGFR3, c-kit, Myb proto-oncogene protein, platelet-derived growth factor receptor beta, vascular endothelial growth factor (VEGF), and Ki-67 was performed. Univariate and multivariate analysis was performed for determination of disease-free survival (DFS) and overall survival (OS).

\section{Results}

In univariate analyses, primary site of nasal cavity and paranasal sinus $(p=0.022)$ and Ki-67 expression of more than 7\% $(p=0.001)$ were statistically significant factors for poor DFS. Regarding OS, perineural invasion ( $p=0.032$ ), high expression of VEGF ( $p=0.033)$, and high expression of Ki-67 ( $p=0.007$ ) were poor prognostic factors. In multivariate analyses, primary site of nasal cavity and paranasal sinus $(p=0.028)$ and high expression of Ki-67 $(p=0.004)$ were independent risk factors for poor DFS, and high expression of VEGF $(p=0.011)$ and Ki-67 ( $p=0.011)$ showed independent association with poor OS.

\section{Conclusion}

High expression of VEGF and Ki-67 were independent poor prognostic factors for OS in ACC.

\section{Key words}

Adenoid cystic carcinoma, Immunohistochemistry, Vascular endothelial growth factor, Ki-67, Prognosis 


\section{Introduction}

Adenoid cystic carcinoma (ACC) is uncommon malignancies, which commonly arise in the salivary glands [1], although nearly half of ACC occurs in other glandular areas, particularly in the nasal cavity and paranasal areas. They also arise in the tongue and the minor salivary glands [2,3], and, very rarely, originate at other sites, such as the external auditory canal, trachea, lung, breast, and Bartholin's glands [2].

ACC is characterized by unpredictable growth and extensive perineural invasion. Typically the natural course of ACC is slow; however, local recurrence and hematogenous spread to the lungs often occur during the course of the disease $[4,5]$. Despite the indolent growth pattern, once metastatic disease is present, disease progression becomes more rapid; onethird of patients die within 2 years of developing multiple metastases [6]. Treatment remains limited to surgery and radiation, and no systemic chemotherapeutic agent has been proven to be effective [7].

Due to its rarity, the molecular biology of ACC has not been well-described [8]. Clinical stage and solid histologic type were poor prognostic indicators of survival in several studies $[9,10]$. In immunohistochemistry, expression of $\mathrm{Myb}$ proto-oncogene protein (MYB), c-kit, vascular endothelial growth factor (VEGF), p53, and Ki-67 are known poor prognostic factors of ACC; however, the relationship between these markers and survival of patients is not well-known [9,11-15]. In addition, there are many potential biomarkers whose significance has not yet been determined.

A previous study reported that fibroblast growth factor (FGF), fibroblast growth factor receptor (FGFR), and plateletderived growth factor receptor beta (PDGFR-beta) represented DNA copy number gain in ACC by microarray-based comparative genomic hybridization [16]. However, there is insufficient information regarding the immunohistochemical results of these markers.

The aim of this study was to evaluate the prognostic value of potential biomarkers related to ACC.

\section{Materials and Methods}

\section{Patients}

The medical records of 188 patients diagnosed with ACC at Seoul National University Hospital between 1990 and 2012 were reviewed. Among these patients, 68 patients who underwent curative surgery and had available tissue were enrolled in this study.

This study was approved by the Institutional Review Board of Seoul National University Hospital (IRB approval No. H-1109-114-379). Demographics, clinical and pathologic data, and treatment-related factors with regard to recurrence and patient survival were collected from medical records. Sixteen patients $(24 \%)$ were followed up until death, and the median follow-up period was 68 months (range, 4 to 263 months).

\section{Immunohistochemistry}

Core tissues ( $2 \mathrm{~mm}$ in diameter) were taken from representative formalin-fixed paraffin-embedded tissue blocks and tissue microarrays were constructed for further immunohistochemical analysis. The antibodies used were basic FGF (bFGF; 1:100, Calbiochem, San Diego, CA), FGFR2 (H2263M01, 1:3,000, Abnova, Taipei, Taiwan), FGFR3 (SC-13121, 1:50, Santa Cruz Biotechnology, Santa Cruz, CA), c-kit (A2502, 1:200, DAKO, Carpinteria, CA), MYB (\#1792-1, 1:80, Epitomics, Burlingame, CA), PDGFR-beta (SC-713, 1:100, Santa Cruz Biotechnology), VEGF (SC-7269, 1:1,000, Santa Cruz Biotechnology), and Ki-67 (M7240, 1:100, DAKO).

Immunostaining was evaluated semi-quantitatively for intensity $(0$, negative; +1 , weak positive; +2 , moderate positive; and +3 , strong positive) and extent $(0,0 \% ;+1,1 \%-25 \%$; $+2,26 \%-50 \% ;+3,51 \%-75 \%$; and $+4,76 \%-100 \%$ ) by two experienced pathologists (S.J.N., Y.K.J.); normal salivary gland samples were used as controls. Score was determined by multiplying extent and intensity. Positivity of expression was defined as a score of 4 or more, and high expression was defined as a score of 8 or more. Conversely, c-kit was graded only by extent $(0$ to +4$)$ because its expression was strong enough in all positive specimens, and positivity was defined as a grade of 2 or more [17].

The immunostained slides for Ki-67 were submitted to virtual microscope scanning under high-power magnification (×200) using ScanScope CS2 eSlide (Aperio Technologies, Vista, CA). Estimation of Ki-67 expression was based on the proportion of positive cells in all tumor cells using the nuclear v9 algorithm of ImageScope software (Aperio Technologies). Ki-67 was considered positive when the tumor cells showed strong nuclear staining intensity.

\section{Statistical analysis}

Statistical analyses of categorical variables were performed using Pearson's $\chi^{2}$ test or Fisher exact probability test where appropriate. The median duration of survival was calculated using the Kaplan-Meier method, and comparisons between groups were made using the log-rank tests. To determine the important prognostic factors, Cox proportional hazards 
Table 1. Clinicopathological features and univariate Cox regression analysis of 68 patients

\begin{tabular}{|c|c|c|c|c|c|}
\hline \multirow{2}{*}{ Characteristic } & \multirow{2}{*}{ No. $(\%)$} & \multicolumn{2}{|c|}{ DFS } & \multicolumn{2}{|c|}{ OS } \\
\hline & & HR & p-value & HR & p-value \\
\hline \multicolumn{6}{|l|}{ Sex } \\
\hline Male & $26(38.2)$ & 1 & & 1 & 0.881 \\
\hline Female & $42(61.8)$ & 0.78 & 0.463 & 1.09 & \\
\hline \multicolumn{6}{|l|}{ Age (yr) } \\
\hline$\leq 45$ & $17(25.0)$ & 1 & & 1 & 0.658 \\
\hline$>45$ & $51(75.0)$ & 1.55 & 0.251 & 1.30 & \\
\hline \multicolumn{6}{|l|}{ Primary site } \\
\hline Salivary gland & $36(52.9)$ & 1 & & 1 & \\
\hline Nasal cavity, paranasal sinus & $16(23.5)$ & 2.34 & 0.022 & 1.61 & 0.420 \\
\hline Tongue, oral cavity & $7(10.3)$ & 2.01 & 0.146 & 0.81 & 0.841 \\
\hline Lung, trachea & $4(5.9)$ & 0.33 & 0.280 & 1.44 & 0.738 \\
\hline Others ${ }^{\text {a) }}$ & $5(7.4)$ & 1.00 & 0.995 & 1.01 & 0.994 \\
\hline \multicolumn{6}{|l|}{ Local treatment } \\
\hline Operation with PORT & 49 (72.1) & 0.96 & 0.919 & 2.16 & 0.311 \\
\hline Operation without PORT & $19(27.9)$ & 1 & & 1 & \\
\hline \multicolumn{6}{|l|}{ Any chemotherapy } \\
\hline Yes & $11(16.2)$ & -b) & -b) & 2.32 & 0.116 \\
\hline No & $57(83.8)$ & - & & 1 & \\
\hline \multicolumn{6}{|l|}{ Perineural invasion } \\
\hline Yes & $23(33.8)$ & 1.26 & 0.501 & 3.05 & 0.032 \\
\hline No & $45(66.2)$ & 1 & & 1 & \\
\hline \multicolumn{6}{|l|}{ Resection margin } \\
\hline Positive & $39(57.4)$ & 0.97 & 0.913 & 1.49 & 0.456 \\
\hline Negative & $29(42.6)$ & 1 & & 1 & \\
\hline
\end{tabular}

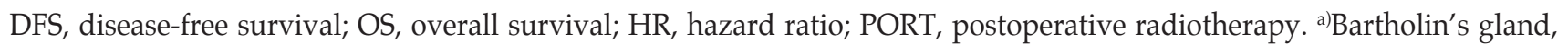
external auditory canal, and lacrimal gland, ${ }^{\mathrm{b}}$ There was no perioperative chemotherapy.

regression models were used in univariate and multivariate analyses. A two-sided value of $p<0.05$ was considered statistically significant. All analyses were performed using SPSS for Windows ver. 20.0 (IBM Co., Armonk, NY).

\section{Results}

\section{Clinicopathological data}

Of the 68 patients, 26 patients were male and 42 were female, with median age of 55 years (range, 26 to 84 years). Primary sites were major salivary glands in 36 cases (53\%), nasal cavity and paranasal sinuses in $16(24 \%)$, oral cavity and tongue in seven (10\%), lung and trachea in four $(6 \%)$, and other sites in five (7\%). Other sites included the lacrimal gland, Bartholin's gland, and external auditory canal. Twenty-three patients (34\%) had perineural invasion and $39(57 \%)$ had involvement of resection margins. Local recurrence was detected in 21 patients $(31 \%)$, regional recurrence in three $(4 \%)$, distant metastases in $35(51 \%)$, and any kind of recurrence in $39(57 \%)$. Of patients with distant metastases, 32 patients $(47 \%)$ had lung metastasis, nine $(13 \%)$ had liver metastasis, six $(9 \%)$ had bone metastasis, and two $(3 \%)$ had central nervous system metastasis.

Analysis of patients was based on clinicopathological factors, including sex, age, primary site, local treatment modality including postoperative radiotherapy, systemic chemotherapy, perineural invasion, and positive resection margins. Survival analysis was performed in each subgroup using the Cox proportional hazards regression model. Among these clinicopathological variables, primary site of nasal cavity and paranasal sinus was a poor prognostic factor for disease-free survival (DFS; hazard ratio [HR], 2.34; 95\% confidence interval $[\mathrm{CI}], 1.13$ to $4.84 ; \mathrm{p}=0.022)$. Perineural invasion was the only significant prognostic factor of poor overall survival (OS; HR, 3.05; 95\% CI, 1.10 to 8.44; $\mathrm{p}=0.032$ ) (Table 1 ). 
Table 2. Positive expression of molecular markers and univariate Cox regression analysis of disease-free survival and overall survival in adenoid cystic carcinoma

\begin{tabular}{|c|c|c|c|c|c|c|c|c|}
\hline \multirow{2}{*}{ Variable } & \multirow{2}{*}{ Score } & \multirow{2}{*}{ No. $(\%)$} & \multicolumn{3}{|c|}{ DFS } & \multicolumn{3}{|c|}{ OS } \\
\hline & & & HR & $95 \% \mathrm{CI}$ & p-value & HR & $95 \% \mathrm{CI}$ & $\overline{\text { p-value }}$ \\
\hline \multirow[t]{2}{*}{ bFGF } & $<4$ & $20(29.4)$ & 1 & & & 1 & & \\
\hline & $\geq 4$ & $48(70.6)$ & 0.76 & 0.39 to 1.49 & 0.424 & 1.25 & 0.35 to 4.47 & 0.737 \\
\hline \multirow{2}{*}{ c-kit } & $<2$ & $16(23.5)$ & 1 & & & 1 & & \\
\hline & $\geq 2$ & $52(76.5)$ & 0.94 & 0.46 to 1.94 & 0.872 & 0.75 & 0.23 to 2.43 & 0.632 \\
\hline \multirow[t]{2}{*}{ FGFR2 } & $<4$ & $62(91.2)$ & 1 & & & 1 & & \\
\hline & $\geq 4$ & $6(8.8)$ & 1.45 & 0.51 to 4.10 & 0.488 & 1.86 & 0.40 to 8.68 & 0.432 \\
\hline \multirow[t]{2}{*}{ FGFR3 } & $<4$ & $64(94.1)$ & 1 & & & 1 & & \\
\hline & $\geq 4$ & $4(5.9)$ & 3.26 & 1.13 to 9.44 & 0.029 & 0.94 & 0.12 to 7.22 & 0.952 \\
\hline \multirow[t]{2}{*}{ MYB } & $<4$ & $44(64.7)$ & 1 & & & 1 & & \\
\hline & $\geq 4$ & $24(35.3)$ & 1.02 & 0.51 to 2.02 & 0.959 & 1.14 & 0.36 to 3.62 & 0.831 \\
\hline \multirow[t]{2}{*}{ PDGFR-beta } & $<4$ & $25(36.8)$ & 1 & & & 1 & & \\
\hline & $\geq 4$ & $43(63.2)$ & 1.10 & 0.57 to 2.13 & 0.770 & 2.43 & 0.68 to 8.62 & 0.171 \\
\hline \multirow[t]{2}{*}{ VEGF } & $<4$ & $6(8.8)$ & 1 & & & 1 & & \\
\hline & $\geq 4$ & 62 (91.2) & 1.96 & 0.60 to 6.40 & 0.264 & 2.53 & 0.33 to 19.39 & 0.371 \\
\hline
\end{tabular}

DFS, disease-free survival; OS, overall survival; HR, hazard ratio; CI, confidence interval; bFGF, basic fibroblast growth factor; FGFR2, fibroblast growth factor receptor 2; FGFR3, fibroblast growth factor receptor 3; MYB, Myb proto-oncogene protein; PDGFR-beta, platelet-derived growth factor receptor beta; VEGF, vascular endothelial growth factor.
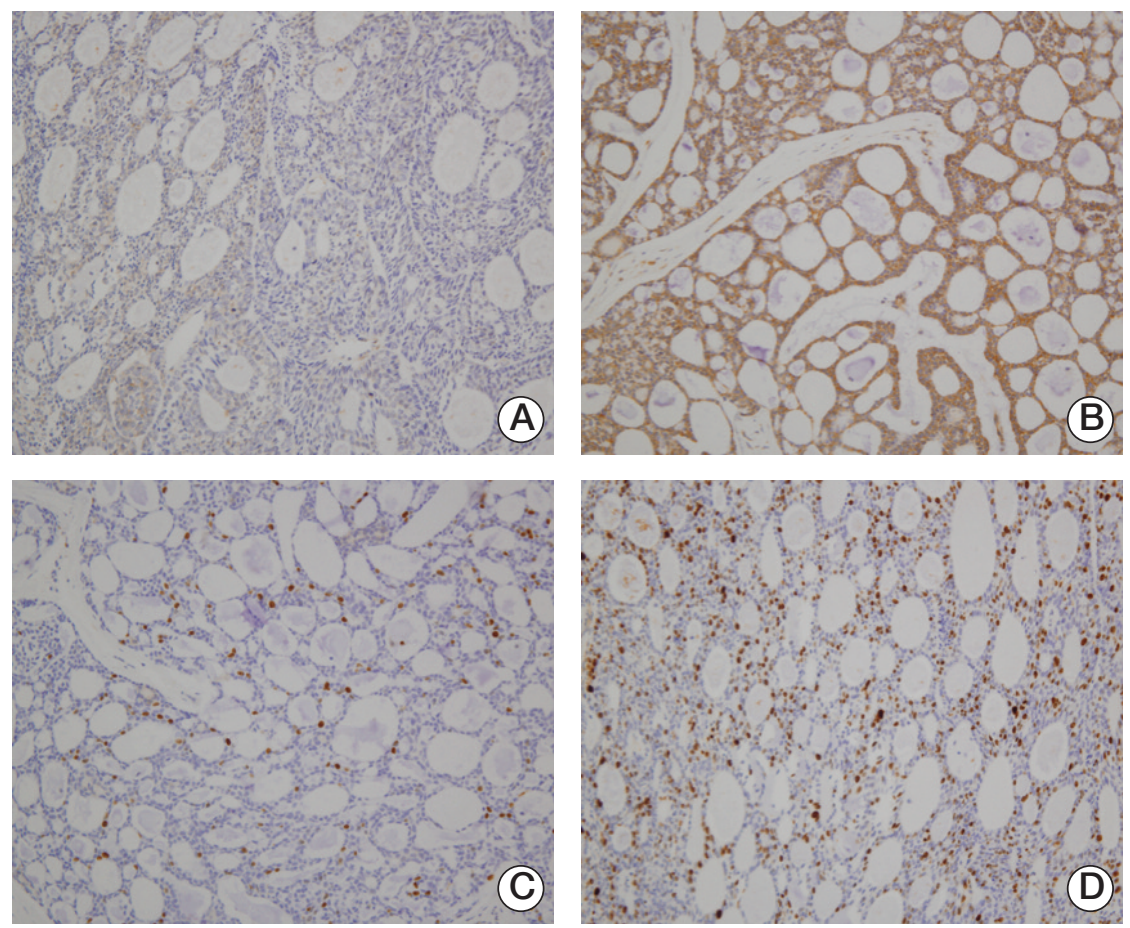

Fig. 1. Immunohistochemical staining of adenoid cystic carcinoma. (A) Low expression of vascular endothelial growth factor (VEGF). (B) High expression of VEGF. (C) Low expression of Ki-67. (D) High expression of Ki-67 (A-D, ×200). 
Table 3. High expression of molecular markers and univariate Cox regression analysis of disease-free survival and overall survival in adenoid cystic carcinoma

\begin{tabular}{|c|c|c|c|c|c|c|c|c|}
\hline \multirow{2}{*}{ Variable } & \multirow{2}{*}{ Score } & \multirow{2}{*}{ No. $(\%)$} & \multicolumn{3}{|c|}{ DFS } & \multicolumn{3}{|c|}{ OS } \\
\hline & & & HR & $95 \% \mathrm{CI}$ & p-value & HR & $95 \% \mathrm{CI}$ & p-value \\
\hline \multirow[t]{2}{*}{ bFGF } & $<8$ & $42(61.8)$ & 1 & & & 1 & & \\
\hline & $\geq 8$ & $26(38.2)$ & 0.85 & 0.44 to 1.62 & 0.618 & 1.25 & 0.44 to 3.51 & 0.673 \\
\hline \multirow[t]{2}{*}{ c-kit } & $<4$ & $51(75.0)$ & 1 & & & 1 & & \\
\hline & $\geq 4$ & $17(25.0)$ & 1.12 & 0.52 to 2.45 & 0.771 & 1.13 & 0.25 to 5.13 & 0.876 \\
\hline \multirow[t]{2}{*}{ FGFR2 } & $<8$ & $67(98.5)$ & 1 & & & 1 & & \\
\hline & $\geq 8$ & $1(1.5)$ & 15.98 & 1.79 to 143.04 & 0.013 & 62.50 & 3.91 to $>99.99$ & 0.003 \\
\hline \multirow[t]{2}{*}{ FGFR3 } & $<8$ & 67 (98.5) & 1 & & & 1 & & \\
\hline & $\geq 8$ & 1 (1.5) & 4.09 & 0.54 to 31.10 & 0.174 & 0.05 & $<0.01$ to $>99.99$ & 0.883 \\
\hline \multirow[t]{2}{*}{ MYB } & $<8$ & $60(88.2)$ & 1 & & & 1 & & \\
\hline & $\geq 8$ & 8 (11.8) & 1.13 & 0.40 to 3.19 & 0.820 & 0.04 & $<0.01$ to 44.54 & 0.370 \\
\hline \multirow[t]{2}{*}{ PDGFR-beta } & $<8$ & $55(80.9)$ & 1 & & & 1 & & \\
\hline & $\geq 8$ & 13 (19.1) & 1.14 & 0.52 to 2.49 & 0.736 & 2.08 & 0.70 to 6.20 & 0.189 \\
\hline \multirow[t]{2}{*}{ VEGF } & $<8$ & $29(42.6)$ & 1 & & & 1 & & \\
\hline & $\geq 8$ & $39(57.4)$ & 1.31 & 0.69 to 2.48 & 0.403 & 3.45 & 1.11 to 10.71 & 0.033 \\
\hline \multirow[t]{2}{*}{ Ki-67 } & $<7 \%$ & $50(73.5)$ & 1 & & & 1 & & \\
\hline & $\geq 7 \%$ & $18(26.5)$ & 3.25 & 1.58 to 6.68 & 0.001 & 4.47 & 1.52 to 13.18 & 0.007 \\
\hline
\end{tabular}

DFS, disease-free survival; OS, overall survival; HR, hazard ratio; CI, confidence interval; bFGF, basic fibroblast growth factor; FGFR2, fibroblast growth factor receptor 2; FGFR3, fibroblast growth factor receptor 3; MYB, Myb proto-oncogene protein; PDGFR-beta, platelet-derived growth factor receptor beta; VEGF, vascular endothelial growth factor.

\section{Immunohistochemical data}

In immunohistochemistry, overexpression of bFGF was observed in 48 patients (71\%), c-kit in $52(77 \%)$, FGFR2 in six (9\%), FGFR3 in four (6\%), MYB in $24(35 \%)$, PDGFR-beta in $43(63 \%)$, and VEGF in $62(91 \%)$. The median Ki-67 value was $4 \%$ (range, $0 \%$ to $33 \%$ ) (Table 2, Fig. 1).

In univariate analysis, positive expression with a score of 4 or more for bFGF, FGFR2, MYB, PDGFR-beta, and VEGF and a score of 2 or more for c-kit did not show correlation with DFS and OS (Table 2). On the other hand, high expression with a score of 8 or more for VEGF was a poor prognostic factor for OS (HR, 3.45; 95\% CI, 1.11 to 10.71; $\mathrm{p}=0.033$ ), and Ki-67 expression of more than 7\% was significant as a poor prognostic factor for both DFS (HR, 3.25; 95\% CI, 1.58 to $6.68 ; \mathrm{p}=0.001)$ and $\mathrm{OS}(\mathrm{HR}, 4.47 ; 95 \% \mathrm{CI}, 1.52$ to 13.18 ; $\mathrm{p}=0.007$ ) (Table 3, Figs. 2, 3A and B). Although a score of 4 or more for FGFR3 and a score of 8 or more for FGFR2 expression also showed a significant $\mathrm{p}$-value, due to the few patients with expression, further validation of the prognostic values is needed in a larger sample size.

In multivariate analysis, primary site of nasal cavity and paranasal sinus (HR, 2.21; 95\% CI, 1.09 to $4.50 ; \mathrm{p}=0.028$ ) and Ki-67 expression (HR, 3.05; 95\% CI, 1.43 to 6.54; $\mathrm{p}=0.004$ ) were independent risk factors for poor DFS. High expression of VEGF (HR, 5.44; 95\% CI, 1.48 to 19.98; $\mathrm{p}=0.011)$ and Ki-67 ( $\mathrm{HR}, 4.83 ; 95 \% \mathrm{CI}, 1.44$ to $16.21 ; \mathrm{p}=0.011)$ were independently significant factors of poor OS. However, perineural invasion was not statistically significant for OS (HR, 2.90; 95\% CI, 1.00 to $8.41 ; \mathrm{p}=0.051$ ) (Table 4). Conduct of further studies to determine relationship between perineural invasion and OS will be needed. Patients who had both high expression of VEGF and Ki-67 expression of more than 7\% showed poorer OS than patients who had high expression of only one of the two proteins (HR, 8.58; 95\% CI, 1.47 to 50.01; $\mathrm{p}=0.017$ ) (Fig. 3C).

\section{Discussion}

The goal of the current study was to identify prognostic markers of ACC by evaluating the relationship between clinicopathological and immunohistochemical data and DFS and OS. The markers bFGF, c-kit, FGFR2, FGFR3, MYB, PDGFRbeta, VEGF, and Ki-67 were evaluated by immunohistochemistry. In univariate analysis, nasal cavity and paranasal sinus as primary site and Ki-67 expression of more than 7\% were risk factors for poor DFS, and presence of perineural 
A

B
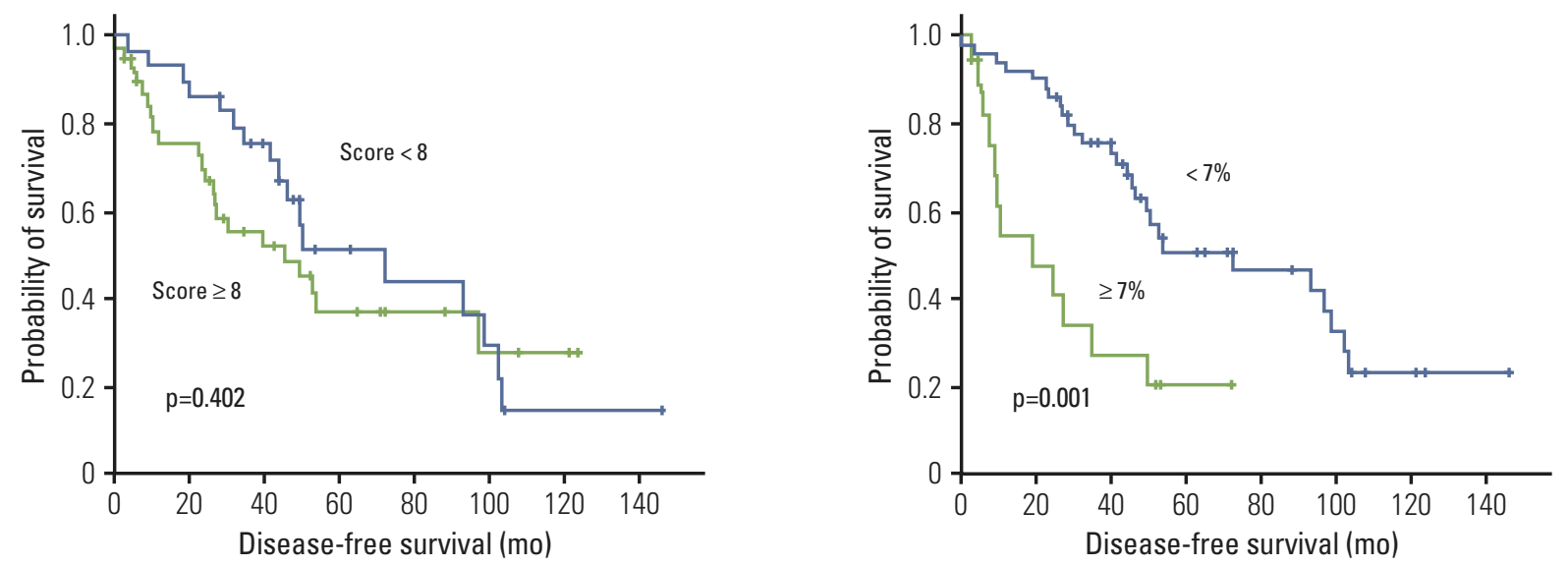

Fig. 2. Kaplan-Meier curve for disease-free survival by vascular endothelial growth factor expression (A) and Ki-67 expression (B). Comparisons were made using the log-rank test.

A

B
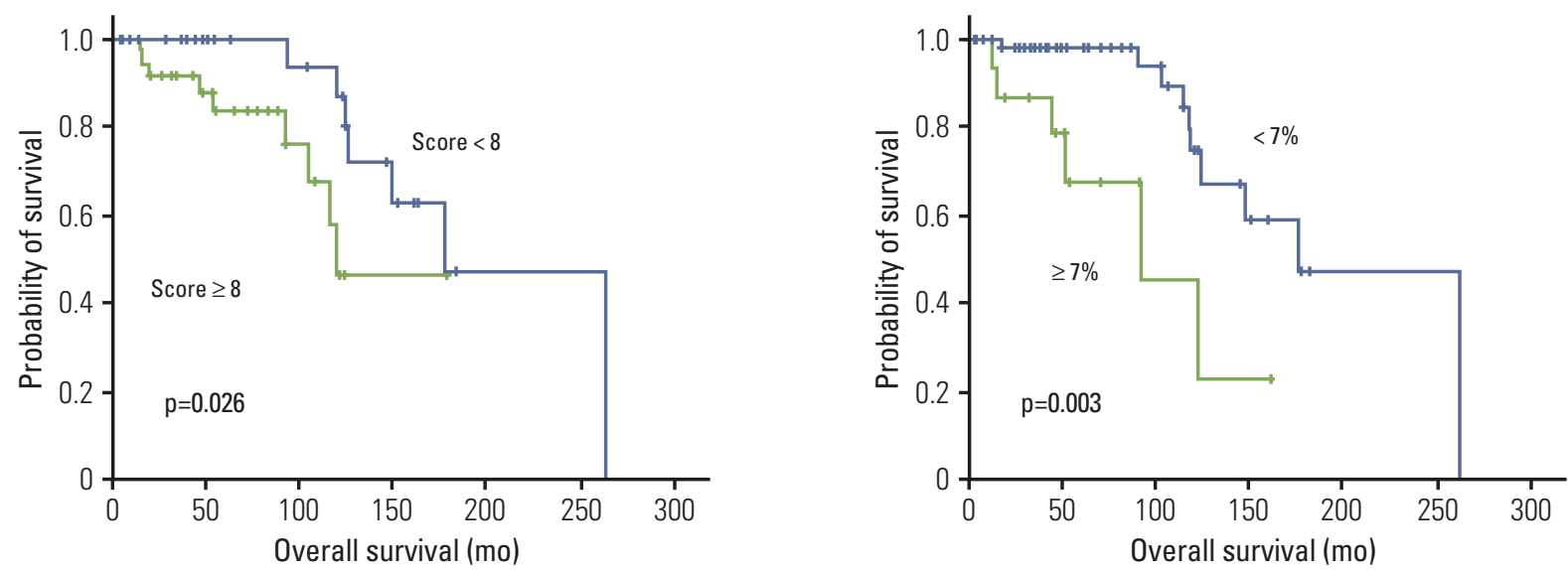

C

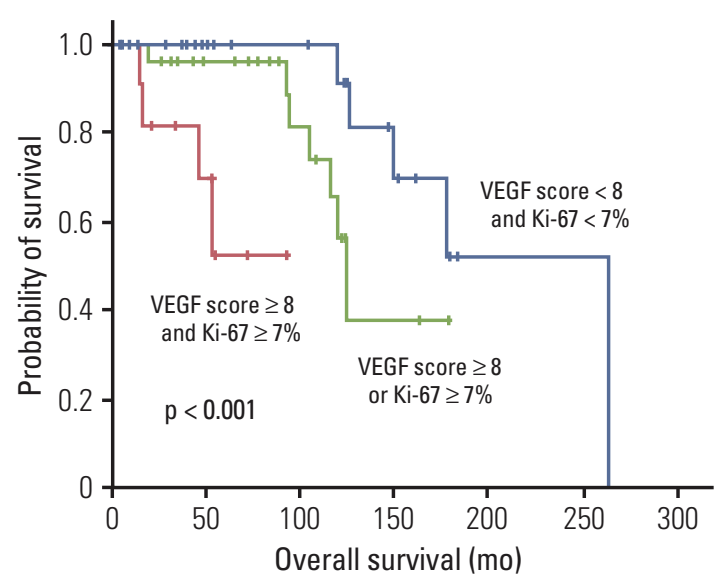

Fig. 3. Kaplan-Meier curve for overall survival by vascular endothelial growth factor (VEGF) expression (A), Ki-67 expression (B), and VEGF and / or Ki-67 expression (C). Comparisons were made using the log-rank test. 
Table 4. Multivariate Cox regression analysis of diseasefree survival and overall survival in adenoid cystic carcinoma

\begin{tabular}{|lllll|}
\hline Variable & Category & HR & $95 \%$ CI & p-value \\
\hline DFS & & & & \\
\hline Nasal area ${ }^{a}$ & No & 1 & & \\
\hline & Yes & 2.21 & 1.09 to 4.50 & 0.028 \\
\hline Perineural invasion & No & 1 & & \\
& Yes & 1.29 & 0.65 to 2.55 & 0.460 \\
\hline Resection margin & No & 1 & & \\
\hline & Yes & 0.84 & 0.39 to 1.78 & 0.641 \\
\hline PORT & No & 1 & & \\
\hline & Yes & 1.09 & 0.47 to 2.52 & 0.837 \\
\hline VEGF & $<8$ & 1 & & \\
\hline & $\geq 8$ & 1.31 & 0.67 to 2.56 & 0.435 \\
\hline Ki-67 & $<7 \%$ & 1 & & \\
\hline \multirow{2}{*}{ OS } & $\geq 7 \%$ & 3.05 & 1.43 to 6.54 & 0.004 \\
\hline Perineural invasion & No & 1 & & \\
\hline & Yes & 2.90 & 1.00 to 8.41 & 0.051 \\
\hline VEGF & $<8$ & 1 & & \\
\hline & $\geq 8$ & 5.44 & 1.48 to 19.98 & 0.011 \\
\hline Ki-67 & $<7 \%$ & 1 & & \\
\hline & $\geq 7 \%$ & 4.83 & 1.44 to 16.21 & 0.011 \\
\hline
\end{tabular}

$\mathrm{HR}$, hazard ratio; $\mathrm{CI}$, confidence interval; DFS, disease-free survival; PORT, postoperative radiotherapy; VEGF, vascular endothelial growth factor; OS, overall survival. ${ }^{\text {a)Pri- }}$ mary sites including nasal cavity and paranasal sinus.

invasion and high expression of VEGF and Ki-67 were significant prognostic factors for poor OS. Among them, high expression of VEGF and Ki-67 were independently significant prognostic factors for OS in multivariate analyses.

ACC has diverse primary sites and there are varying levels of difficulty of operation according to primary sites. Ciccolallo et al. [18], who analyzed survival of ACC in a large population using the EUROCARE database, showed that ACC originating from nasal cavity, pharynx, and larynx have poorer 5-year survival rate than oral cavity and major salivary glands. In the current study, although no significant difference of OS was observed among primary sites, nasal cavity and paranasal sinus were the sites showing poor DFS.

In the current study, perineural invasion was observed in 23 patients (34\%), and it was the only significant prognostic factor for OS among the clinicopathological values evaluated. Its importance as a poor prognostic factor is well-established in the literature $[5,9,19]$. In the study reported by Garden et al. [19], in addition to the presence of perineural invasion, positive resection margin was an adverse prognostic factor in terms of local control, which was not the case in this study.

VEGF, a critical factor in tumor angiogenesis, has been widely assessed in various types of cancer. Association of VEGF expression with poor prognosis of ACC has been reported in a few studies $[12,13,20]$. In an analysis with 29 patients of salivary gland carcinomas, Lim et al. [13] reported a significant association of high expression of VEGF with poor OS. However, only 15 patients were ACC patients and others were mucoepidermoid carcinomas. Li et al. [20] reported correlation of high VEGF expression with tumor stage in 55 patients of salivary ACCs, but not with local recurrence and survival rate. High expression of VEGF was an independently important factor for poor OS in the current study. Although VEGF expression showed no statistically significant difference for DFS, it showed a poor trend in the Kaplan-Meier curve. Conduct of further studies with a large population will be needed in order to verify correlation of VEGF expression with poor DFS.

Ki-67 is associated with cellular proliferation in tumor progression. Several studies identified high expression of Ki-67 as a negative prognostic factor in salivary gland carcinomas $[13,14,21]$. Nordgard et al. [21], who analyzed 44 patients diagnosed with ACCs, reported a significant difference of poor DFS with Ki-67 expression of more than $4 \%$. Lim et al. [13], who analyzed survival of 29 patients, 15 of salivary ACCs and 14 of mucopeidermoid carcinomas, reported correlation between high expression of $\mathrm{Ki}-67$ and poor OS. In addition, Ettl et al. [14] demonstrated relationship between Ki-67 expression and poor OS in 101 patients of salivary gland carcinomas. However, that population only included 25 ACC patients. In the current study, Ki-67 was the strongest prognostic marker for both poor DFS and OS in ACC. This is the largest study to date composed only of ACC patients, and proves significance of Ki-67 expression. In our analysis a cut-off value of $7 \%$ showed the greatest significance in OS.

One of the most studied biomarkers of ACC is the translocation between $M Y B$ oncogene and NFIB translocation factor. Approximately $50 \%$ of patients have a MYB-NFIB translocation, and these patients tend to have a higher risk for local relapse [22]. MYB over-expression has been associated with $M Y B-N F I B$ translocation, although its relationship with ACC prognosis was uncertain [23]. In our study, MYB expression was not a significant factor in predicting poor DFS or OS. Another biomarker, c-kit, has been the subject of several research studies in ACC. In a recent study, c-kit expression showed correlation with clinical stage, perineural invasion, locoregional recurrence, and distant metastases; however, there was little data regarding the relationship between c-kit expression and OS [11]. c-kit expression was not a significant prognostic factor of DFS and OS in the current study.

PDGFR-beta expression is known to be a poor prognostic 
factor in breast cancer $[24,25]$. Similarly, DNA copy numbers of PDGFR-beta on chromosome 5 are increased in ACC and it is a possible factor contributing to the progression of ACC [16]. However, the relationship between PDGFR-beta expression and the prognosis of ACC has not been previously reported. The current study showed that high expression of PDGFR-beta was not significant as a prognostic factor in ACC. DNA copy number gain of FGF and FGFR in ACC was also reported in an earlier study [16]; however, there was almost no data on the immunohistochemical expression of FGF and FGFR which was investigated in this study. bFGF expression of more than $70 \%$ was observed, however expression of FGFR2 and FGFR3 was rare. Among them, bFGF had no significant value as prognostic markers, and conduct of further studies on FGFR2 and FGFR3 will be needed in order to provide patients with practical recommendations.

Most studies conducted in the past analyzed the utility of immunohistochemical markers with respect to ACC invasiveness and recurrence. However, the current study was conducted in a large population and investigated the correlation between several immunohistochemical markers and survival, which is more critical for ACC prognosis. We acknowledge that there are some limitations to this study, including the lack of validating the immunohistochemical cut-off values used, and the fact that this is a retrospective study.

\section{Conclusion}

In conclusion, high expression of VEGF and high expression of Ki-67 are independent prognostic factors of poor OS in ACC. Therefore, more aggressive and differentiated treatment should be provided to patients who show high expression of these markers. Conduct of further prospective studies in larger populations will be necessary in order to confirm these prognostic factors and to elucidate appropriate treatment modalities.

\section{Conflicts of Interest}

Conflict of interest relevant to this article was not reported.

\section{Acknowledgments}

This study was supported by SNUH Research Fund (grant no. 04-2013-0760 and 30-2013-0070). We especially thank Ju Yon Kim for her data management.

\section{References}

1. Renehan A, Gleave EN, Hancock BD, Smith P, McGurk M. Long-term follow-up of over 1000 patients with salivary gland tumours treated in a single centre. Br J Surg. 1996;83:1750-4.

2. Gondivkar SM, Gadbail AR, Chole R, Parikh RV. Adenoid cystic carcinoma: a rare clinical entity and literature review. Oral Oncol. 2011;47:231-6.

3. Spiro RH. Salivary neoplasms: overview of a 35-year experience with 2,807 patients. Head Neck Surg. 1986;8:177-84.

4. Sung MW, Kim KH, Kim JW, Min YG, Seong WJ, Roh JL, et al. Clinicopathologic predictors and impact of distant metastasis from adenoid cystic carcinoma of the head and neck. Arch Otolaryngol Head Neck Surg. 2003;129:1193-7.

5. Huang M, Ma D, Sun K, Yu G, Guo C, Gao F. Factors influencing survival rate in adenoid cystic carcinoma of the salivary glands. Int J Oral Maxillofac Surg. 1997;26:435-9.

6. Spiro RH. Distant metastasis in adenoid cystic carcinoma of salivary origin. Am J Surg. 1997;174:495-8.

7. Laurie SA, Ho AL, Fury MG, Sherman E, Pfister DG. Systemic therapy in the management of metastatic or locally recurrent adenoid cystic carcinoma of the salivary glands: a systematic review. Lancet Oncol. 2011;12:815-24.
8. Liu J, Shao C, Tan ML, Mu D, Ferris RL, Ha PK. Molecular biology of adenoid cystic carcinoma. Head Neck. 2012;34: 1665-77.

9. da Cruz Perez DE, de Abreu Alves F, Nobuko Nishimoto I, de Almeida OP, Kowalski LP. Prognostic factors in head and neck adenoid cystic carcinoma. Oral Oncol. 2006;42:139-46.

10. Khan AJ, DiGiovanna MP, Ross DA, Sasaki CT, Carter D, Son $\mathrm{YH}$, et al. Adenoid cystic carcinoma: a retrospective clinical review. Int J Cancer. 2001;96:149-58.

11. Tang $Y$, Liang $X$, Zheng $M$, Zhu Z, Zhu G, Yang J, et al. Expression of c-kit and Slug correlates with invasion and metastasis of salivary adenoid cystic carcinoma. Oral Oncol. 2010;46:311-6.

12. Yu F, Jiang XZ, Chen WT, Zhao YF, Zhou XJ. Microvessel density and expression of vascular endothelial growth factor in adenoid cystic carcinoma of salivary gland. Shanghai Kou Qiang Yi Xue. 2003;12:443-6.

13. Lim JJ, Kang S, Lee MR, Pai HK, Yoon HJ, Lee JI, et al. Expression of vascular endothelial growth factor in salivary gland carcinomas and its relation to p53, Ki-67 and prognosis. J Oral Pathol Med. 2003;32:552-61. 
14. Ettl T, Schwarz S, Kleinsasser N, Hartmann A, Reichert TE, Driemel O. Overexpression of EGFR and absence of C-KIT expression correlate with poor prognosis in salivary gland carcinomas. Histopathology. 2008;53:567-77.

15. Zhu QR, White FH, Tipoe GL. p53 oncoprotein accumulation in adenoid cystic carcinoma of parotid and palatine salivary glands. Pathology. 1997;29:154-8.

16. Vekony H, Ylstra B, Wilting SM, Meijer GA, van de Wiel MA, Leemans CR, et al. DNA copy number gains at loci of growth factors and their receptors in salivary gland adenoid cystic carcinoma. Clin Cancer Res. 2007;13:3133-9.

17. Salehinejad J, Mohtasham N, Bagherpour A, AbbaszadehBidokhty H, Ghazi A. Evaluation of c-kit protein (CD117) expression in common salivary gland neoplasms. J Oral Maxillofac Pathol. 2014;18:177-82.

18. Ciccolallo L, Licitra L, Gatta G; EUROCARE Working Group. Gatta G. Survival from salivary glands adenoid cystic carcinoma in European populations. Oral Oncol. 2009;45:669-74.

19. Garden AS, Weber RS, Morrison WH, Ang KK, Peters LJ. The influence of positive margins and nerve invasion in adenoid cystic carcinoma of the head and neck treated with surgery and radiation. Int J Radiat Oncol Biol Phys. 1995;32:619-26.

20. Li Z, Tang P, Xu Z. Clinico-pathological significance of microvessel density and vascular endothelial growth factor expression in adenoid cystic carcinoma of salivary glands. Zhonghua Kou Qiang Yi Xue Za Zhi. 2001;36:212-4.

21. Nordgard S, Franzen G, Boysen M, Halvorsen TB. Ki-67 as a prognostic marker in adenoid cystic carcinoma assessed with the monoclonal antibody MIB1 in paraffin sections. Laryngoscope. 1997;107:531-6.

22. West RB, Kong C, Clarke N, Gilks T, Lipsick JS, Cao H, et al. MYB expression and translocation in adenoid cystic carcinomas and other salivary gland tumors with clinicopathologic correlation. Am J Surg Pathol. 2011;35:92-9.

23. Mitani Y, Li J, Rao PH, Zhao YJ, Bell D, Lippman SM, et al. Comprehensive analysis of the MYB-NFIB gene fusion in salivary adenoid cystic carcinoma: incidence, variability, and clinicopathologic significance. Clin Cancer Res. 2010;16:4722-31.

24. Paulsson J, Sjoblom T, Micke P, Ponten F, Landberg G, Heldin $\mathrm{CH}$, et al. Prognostic significance of stromal platelet-derived growth factor beta-receptor expression in human breast cancer. Am J Pathol. 2009;175:334-41.

25. Koo JS, Park S, Kim SI, Lee S, Park BW. The impact of caveolin protein expression in tumor stroma on prognosis of breast cancer. Tumour Biol. 2011;32:787-99. 